\title{
ORIGINAL
}

\section{INFLUENCIA DE LAS DESIGUALDADES SOCIALES, LA CONFLICTIVIDAD SOCIAL Y LA POBREZA EXTREMA SOBRE LA MORBILIDAD POR TUBERCULOSIS EN LA CIUDAD DE BARCELONA (*)}

Estela Díaz de Quijano (1), M. Teresa Brugal (1), M. ${ }^{a}$ Isabel Pasarín (2), Hernando Galdós-Tangüís (1)(3), Joan Caylà (1)(3) y Carme Borrell (2).

(1) Servei d'Epidemiologia. Institut Municipal de Salut Pública de Barcelona.

(2) Servei d'Informació Sanitària. Institut Municipal de Salut Pública de Barcelona.

(3) Unidad de Investigación en Tuberculosis. Barcelona.

(*) Estudio parcialmente financiado por el Fondo de Investigaciones Sanitarias (Expediente núm 96/0824).

\section{RESUMEN}

Fundamentos: El objetivo principal del presente estudio es analizar las desigualdades de la incidencia de co-enfermedad tuberculosis/drogadicción y de la incidencia de tuberculosis sin drogadicción en los barrios de Barcelona y su asociación con indicadores socioeconómicos y de privación social y material, durante los años 1990-1995.

Métodos: Estudio ecológico que incluyó los casos de tuberculosis en personas residentes en la ciudad de Barcelona, siendo el barrio la unidad de análisis. Se consideró a los usuarios de drogas por vía intravenosa de 15 a 49 años y a los no usuarios de drogas por vía intravenosa mayores de 14 años. Se utilizó la tasa de incidencia anual media de tuberculosis estandarizada por edad. Como indicadores sociales se consideraron: el fenómeno de zona centro, el desempleo, la instrucción insuficiente, el hacinamiento, la conflictividad social y la pobreza extrema. En el análisis bivariado la asociación entre la incidencia de tuberculosis y los indicadores sociales se estudió mediante el coeficiente de correlación de Spearman, y en el análisis multivariado mediante la regresión de Poisson.

Resultados: Las tasas de incidencia fueron más elevadas en los barrios del centro de la ciudad, seguido de barrios obreros periféricos de más reciente construcción en los dos colectivos estudiados, y de otros barrios periféricos antiguos en los no usuarios de drogas por vía intravenosa. En el colectivo de usuarios de drogas por vía intravenosa, las variables asociadas a un mayor riesgo de tuberculosis fueron: ser hombre (RR=7,42, IC 95\%: 6,16-8,93), la edad $(\mathrm{RR}=61,51$, IC 95\%: 34,64-109,20), el desempleo ( RR $=1,68$, intervalo de confianza al $95 \%$ - IC95\%-: $1,51-1,88)$, la conflictividad social ( $R R=1,29$, IC $95 \%$ : 1,04-1,58), el hacinamiento ( $R R=1,36$, IC 95\%: 1,19-1,55) y el fenómeno de zona centro (RR=1,92, IC 95\% 1,48-2,50). En los hombres no usuarios de drogas por vía intravenosa se detectó una interacción entre desempleo y edad, y los barrios con pobreza extrema y los barrios céntricos presentaron un riesgo de tuberculosis superior $(\mathrm{RR}=1,11$, IC $95 \% 1,08-1,135$ y $R R=1,80$, IC95\%: 1,51-2,14). En las mujeres no usuarias de drogas por vía intravenosa, se asoció la menor edad ( $R R=1,29$, IC 95\%: 1,05-1,57), el desempleo ( $R R=1,18$, IC 95\%: $1,09-1,26)$, la pobreza extrema ( $R R=1,95$, IC $95 \%: 1,56-2,45)$ y el fenómeno zona centro ( $R R=1,72$, IC 95\%: 1,35-2,19).

\section{Correspondencia:}

Estela Díaz de Quijano

Institut Municipal de Salut Pública

Pl. Lesseps 1

08023 Barcelona

Correo electrónico: estela@imsb.bcn.es

\section{ABSTRACT}

The Influence of Social Inequalities, Social Unrest and Extreme Poverty on Tuberculosis Morbidity in the City of Barcelona

Background: The main purpose of this study was to analyse inequalities between tuberculosis/intravenous drug user and tuberculosis/not intravenous drug user among the different neighbourhoods of Barcelona and the relationship to socioeconomic indicators and social / material deprivation indicators throughout the 1990-1995 period.

Methods: An ecological study which included all cases of tuberculosis among residents of Barcelona, the analysis unit being the neighbourhood. Intravenous drug users within the 15-49 age range and non-intravenous drug users over age 14 were included. The mean annual incidence rate standardized by age was used. The social indicators considered were the inner city areas, unemployment, less than primary education, overcrowding, social unrest and extreme poverty. In the bivariate analysis, the relationship between tuberculosis incidence and the social indicators was studied by means of the Spearman correlation coefficient, and the multivariate analysis by means of the Poisson regression.

Results: The incidence rates were higher in the inner city neighbourhoods, followed by the newer outlying working class neighbourhoods in both groups studied, and in other older outlying districts for the non-users of intravenous drugs. Among the intravenous drug user group, the variables related to a greater risk of tuberculosis were: $\operatorname{man}(\mathrm{RR}=7.42$, confidence interval at 95\% ICI95\%-: 6.16 - 8.93), age (RR=61.51, CI95\%: 34.64 . $109.20)$, unemployment $(\mathrm{RR}=1.68$, C195\%: $1.51-1.88)$, social unrest $(\mathrm{RR}=1.29$, CI 95\%: 1. 04-1.58), overcrowding ( $\mathrm{RR}=1.36$, CI $95 \%: 1.19-1.55)$ and inner city areas ( $R R=1.92$, CI $95 \%$ $1.48-2.50)$. Among the male non-users of intravenous drugs, no interaction between unemployment and age was found. Neighbourhoods with extreme poverty and inner city neighbourhoods showed a higher risk of tuberculosis ( $R R=1.11$, CI 95\% $1.08-1.135$ and $R R=1.80$, CI95\%: 1.51-2.14). Among the female non-users of intravenous drugs, a relationship was found in those of lesser age ( $R R=1.29$, CI 95\%: 1.05-1.57), unemployment $(\mathrm{RR}=1.18$, CI $95 \%: 1.09-1.26)$, extreme poverty $(\mathrm{RR}=1.95, \mathrm{CI}$ 95\%: $1.56-2.45)$ and inner city areas $(\mathrm{RR}=1.72$, CI 95\%: 1.35-2.19). 
Conclusión: Las desigualdades en la distribución de la tuberculosis están relacionadas con distintos indicadores sociales como el desempleo y los barrios de zona centro, y son diferentes en los dos colectivos estudiados, relacionándose con la conflictividad social y el hacinamiento en los usuarios de drogas por vía intravenosa y con la pobreza extrema en los no usuarios de drogas por vía intravenosa.

Palabras clave: Desigualdades en salud. Tuberculosis Drogadicción. Clase Social. Incidencia.

\section{INTRODUCCIÓN}

En los últimos años la naturaleza y magnitud de la tuberculosis (TB) ha cambiado tanto en los países pobres como en los ricos ${ }^{1}$ debido sobre todo a la infección por $\mathrm{VIH}^{2,3}$ $\mathrm{y}$, en nuestro medio, debido también al abuso de drogas por vía intravenosa ${ }^{4}$. En Barcelona, las personas infectadas por VIH entre los años 1987 y 1990 sólo representaban el $20 \%$ de todos los casos de TB. A partir de 1991 este porcentaje empezó a incrementarse hasta llegar al $29 \%$ en 19945 .

Esta relación entre el resurgimiento de la TB y el VIH destaca sobre todo en las zonas del centro de las grandes ciudades deprimidas socioeconómicamente (inner cities o fenómeno zona centro), en donde se combinan la concentración de usuarios de drogas, las condiciones de vida precarias y la infección por VIH, que ha tenido efectos devastadores sobre la población juvenil ${ }^{6}$. Según este fenómeno de zona centro, la existencia de grupos minoritarios con procesos de marginación dentro de un área de una gran ciudad puede afectar la integración social de toda la zona y conllevará importantes problemas socioeconómicos y culturales?

En general, la privación socioeconómica de la población se ha relacionado con un peor estado de salud y con un amplio espectro de patologías ${ }^{8,9}$, más concretamente, tanto el abuso de drogas como la TB tienen como factores determinantes la privación social y económica ${ }^{10-14}$. En países con baja prevalencia de infección tuberculosa, como el Reino Unido, la infección por el $\mathrm{VIH}$ ha tenido poco impacto sobre la TB pero, en cambio, factores como la inmigración procedente de países pobres, el hacinamiento y la privación
Conclusion: Inequalities in tuberculosis are related to different social indicators such as unemployment and inner city areas. A difference exists between the two groups studied. Tuberculosis is related with social unrest and overcrowding among intravenous drug users and with extreme poverty among non-users of intravenous drugs.

Keywords: Social inequalities in health. Tuberculosis. Drug addiction. Social class.

socioeconómica (pobreza, desempleo y personas «sin techo») se han relacionado con el aumento de la enfermedad ${ }^{15-18}$. Algunos estudios han relacionado la TB con el hacinamiento ${ }^{19}$ y destacan la importancia de la TB en la población «sin techo» $»^{2,20-22}$.

En Barcelona concretamente, existen importantes desigualdades en mortalidad en las diferentes áreas geográficas que están relacionadas con el nivel socioeconómico ${ }^{23-25}$, agravadas por el fenómeno de zona centro que se da en los barrios del centro de la ciudad, los cuales aglutinan los peores indicadores socioeconómicos, así como una elevada morbi-mortalidad, tanto por tuberculosis como por infección por VIH y abuso de dro$\operatorname{gas}^{26}$.

El objetivo principal del presente estudio es analizar las desigualdades de la incidencia de co-enfermedad tuberculosis/drogadicción y de la incidencia de tuberculosis sin drogadicción en los barrios de Barcelona y su asociación con indicadores socioeconómicos y de privación social y material, durante los años 1990-1995. Como objetivo secundario, se validó la unidad geográfica utilizada (barrio de residencia) del registro de $\mathrm{TB}$ de la ciudad de Barcelona, en el año 1995.

\section{SUJETOS Y MÉTODO}

\section{Desigualdades sociales por TB}

La población de estudio estuvo constituida por las personas diagnosticadas de TB residentes en la ciudad de Barcelona, identificadas entre 1990 y 1995 a partir del registro de casos del Programa de TB de Barcelona, 
cuya exhaustividad es superior al $95 \%$ debido a que se basa en una vigilancia activa ${ }^{5}$.

Se diseñó un estudio transversal ecológico utilizando el barrio como unidad de análisis. La población en los 38 barrios de la ciudad oscilaba entre 1.524 y 109.751 habitantes en $1991^{27}$. Cabe señalar que se utilizó este tipo de diseño debido a que las variables socioeconómicas del registro de casos de TB no están suficientemente cumplimentadas para poder hacer un estudio de base individual.

Las variables individuales procedentes del registro de TB fueron: el sexo, la edad, el barrio de residencia y el tipo de colectivo. Se consideraron dos colectivos según prácticas de riesgo, por una parte el de usuarios de drogas por vía intravenosa (UDIs) de 15 a 49 años (casos con co-enfermedad tuberculosis/drogadicción) y por otra, el resto de individuos de 15 años o más (no UDIs, que son los casos de tuberculosis sin drogadicción). Los grupos de edad utilizados fueron de 15 a 24 años y de 25 a 49 años en los UDIs, y de 15 a 34 años y mayores de 34 en los no UDIs. Esta estratificación diferencial se realizó debido a que la población de UDIs es más joven que el resto de pacientes, siendo el rango de edad mucho menor.

Las fuentes de información utilizadas para obtener los datos de población de hecho y los indicadores socioeconómicos fueron el Padrón Municipal de Habitantes de la ciudad de Barcelona de 199127, el Censo de Población y Vivienda de $1991^{28}$, el Registro de Pensiones Mínimas de reinserción de los años 1994, 1995 y 1996, y los ingresos preventivos en la prisión de hombres del año 1995. Los datos de estos dos últimos registros se obtuvieron de forma específica para el presente estudio.

Los indicadores de salud utilizados para el análisis descriptivo y bivariado fueron las tasas de incidencia anual media de TB de UDIs y de TB de personas que no son UDIs, ambas respecto a la población general, estandarizadas por edad por 100.000 habitan- tes (TEI), calculada para cada barrio entre 1990 y 1995.

Como indicadores socioeconómicos se utilizaron las variables ecológicas disponibles de cada barrio: desempleo masculino y femenino (porcentaje de personas que se declararon desempleadas en el censo de 1991), la instrucción insuficiente masculina y femenina (porcentaje de personas mayores de 16 años analfabetas o con una titulación inferior a los estudios primarios según el padrón de 1991), y el hacinamiento (porcentaje de viviendas del barrio en que la razón entre el número de convivientes y el número de habitaciones era superior a 1, según el censo de 1991). Se utilizó como indicador de conflictividad social la tasa de ingresos en prisión preventiva de hombres mayores de 21 años por 100.000 habitantes en el año 1995, y como indicador de pobreza extrema la tasa de pensiones mínimas de reinserción por 1.000 habitantes. Como indicador del fenómeno de zona centro, se utilizó el conjunto de los 3 barrios con indicadores más desfavorables, ubicados en el centro histórico de la ciudad.

Las tasas de incidencia de TB se estandarizaron por edad (utilizando grupos de 10 años) según el método directo ${ }^{29}$, siendo la población de referencia la de Barcelona de 1991. Los indicadores sociales de desempleo, nivel de instrucción y conflictividad social también se estandarizaron por edad (utilizando grupos de 10 años) según el método directo con la misma población de referencia $^{30}$. El indicador de pobreza extrema no se pudo estandarizar por edad, ya que no se disponía de los datos necesarios.

En el análisis bivariado la asociación entre los indicadores sociales y la TEI se estudió mediante el coeficiente de correlación de Spearman. Previamente al análisis multivariado, se realizó un análisis de la relación entre la tasa de incidencia de TB y cada una de las variables sociales en los diferentes estratos de edad y sexo, con el fin de categorizar las variables que cumplían los criterios de li- 
nealidad, como fueron la pobreza extrema y la conflictividad social. De esta forma, se crearon dos categorías para el indicador de conflictividad social (barrio con tasas de más de 400 hombres encarcelados por 100.000 habitantes), y para el de pobreza extrema (barrios con tasas superiores a 3 pensiones mínimas de reinserción por 1.000 habitantes). En el análisis multivariado se ajustaron modelos de regresión de Poisson para obtener medidas de riesgo (Riesgo Relativo con un intervalo de confianza al 95\%, RR $\mathrm{IC}_{95 \%}$ ), siendo la variable dependiente las tasas de incidencia de TB en cada barrio y como variables independientes se incluyeron la edad agrupada, los indicadores ecológicos sin estandarizar por edad y las interacciones relevantes a nivel conceptual teniendo en cuenta las asociaciones estadísticamente significativas en el análisis bivariado y las distribuciones de las variables en los distintos estratos de edad y sexo. Para los indicadores que no cumplían los criterios de linealidad, se calculó el RR al $\mathrm{IC}_{95 \%}$ para incrementos del 3\% de desempleo y para incrementos del $10 \%$ de hacinamiento.

No se incluyeron en el mismo modelo las variables que presentaban colinealidad como por ejemplo el desempleo y la instrucción insuficiente. La bondad de ajuste del modelo se obtuvo mediante la lejanía (deviance) ${ }^{31}$. Se realizaron análisis separados para cada colectivo y para cada sexo. Se eliminó un barrio del análisis porque, debido a sus características demográficas (sólo tenía 1.524 habitantes), presentaba TEIs con valores extremos inestables que distorsionaban los resultados de los análisis. El análisis estadístico bivariado se realizó con el paquete de programas estadísticos del SPSS $^{32}$ y el multivariado con el programa EGRET $^{33}$.

\section{Validación de la variable barrio de residencia}

La población de estudio fueron todos los casos de TB residentes en la ciudad de Barcelona declarados durante el año 1995. Se consideró válida la variable barrio de residencia que consta en el registro de tuberculosis, que es el barrio declarado por el paciente, debido a que procede de un sistema de vigilancia activa, y se acerca por tanto a la realidad de forma homogénea en toda la ciudad. Así pues, se utilizó el barrio declarado en el registro de TB, y se comparó con el que figuraba en el Padrón de Habitantes de 1991. La comparación de la proporción de casos concordantes en las diferentes zonas geográficas se realizó mediante la prueba de Ji-cuadrado.

\section{RESULTADOS}

\section{Desigualdades sociales por TB}

Durante los seis años del estudio se detectaron 5.335 casos de TB, de los que 3.835 $(72 \%)$ eran hombres y 1.500 mujeres (28\%); 1.151 eran UDIs $(21,6 \%)$ y el resto no UDIs (tabla 1). En los diferentes barrios el rango de casos de TB osciló entre 0 y 138 UDIs, y entre 4 y 463 no UDIs. En los UDIs la edad media de los hombres fue de 31,3 (con una desviación estándar $\mathrm{DE}=5,4$ ) y la de las mujeres de 29,3 ( $\mathrm{DE}=4,6)$. En los no UDIs los hombres tuvieron una edad media de 45,8 (DE=17,9) y las mujeres de 42,4 $(\mathrm{DE}=20,6)$. La TEI de TB que son UDIs para el total de Barcelona se situó en 24,5

\section{Tabla 1}

Distribución por edad y sexo de los casos de tuberculosis en usuarios de drogas por vía intravenosa (UDIs) y no UDIs, Barcelona 1990-1995

\begin{tabular}{|lrrrr|}
\hline & \multicolumn{2}{c}{ UDI } & \multicolumn{2}{c|}{ No UDI } \\
\cline { 2 - 5 } & $N$ & $(\%)$ & \multicolumn{1}{c|}{$N$} & $(\%)$ \\
\hline $\begin{array}{l}\text { Grupos de edad } \\
\text { Jóvenes * } \\
\text { Mayores ** }\end{array}$ & 1052 & $(91,4)$ & 2649 & $(63,3)$ \\
Sexo & & & & \\
$\quad$ Hombres & 952 & $(82,7)$ & 2883 & $(68,9)$ \\
Mujeres & 199 & $(17,3)$ & 1301 & $(31,1)$ \\
\hline Total & 1151 & $(100)$ & 4184 & $(100)$ \\
\hline
\end{tabular}

* Jóvenes UDI: 15-24 años; jóvenes no UDI: 15-34 años.

** Mayores UDI: 25-49 años; mayores no UDI: > 35 años. 
por 100.000 habitantes de 15 a 49 años, $y$, la de los TB que no son UDIs, en 50,9 por 100.000 habitantes mayores de 14 años.

Las TEI de TB, tanto de personas que son UDIs como que no son UDIs, fueron más elevadas en los cuatro barrios del centro histórico de la ciudad (casco antiguo), seguido, en los dos colectivos, de barrios obreros de la periferia de más reciente construcción y de otros barrios periféricos antiguos en los no UDIs. Por el contrario, las incidencias más bajas se dieron en los barrios más acomodados de la ciudad.

Tal como se muestra en la tabla 2 y en la figura 1, la mayoría de correlaciones bivariadas fueron estadísticamente significativas. En el colectivo de los UDIs, los indicadores de situación socioeconómica desfavorable (desempleo e instrucción insuficiente) se hallan muy correlacionados con la incidencia de TB, presentando un coeficiente de correlación de Spearman $\left(\mathrm{r}_{\mathrm{s}}\right)$ de 0,82 y 0,79 respectivamente. En el colectivo de los no UDIs la incidencia de TB está fuertemente

Tabla 2

Coeficiente de correlación de Spearman entre la tasa de incidencia de tuberculosis estandarizada por edad y los indicadores sociales en los barrios según sexo. Colectivos de UDIs y no UDIs. Barcelona 1990-1995

\begin{tabular}{|c|c|c|c|}
\hline & Hombres & Mujeres & Total \\
\hline \multicolumn{4}{|l|}{ UDI } \\
\hline Desempleo $^{1}$ & $0,81^{*}$ & $0,62 *$ & $0,82 *$ \\
\hline Instrucción Insuficiente $^{1}$ & $0,76^{*}$ & $0,58^{*}$ & $0,79 *$ \\
\hline Pobreza Extrema & $0,68^{*}$ & $0,62 *$ & $0,70^{*}$ \\
\hline Conflictividad social $^{1}$ & $0,74^{*}$ & $0,52 *$ & $0,73^{*}$ \\
\hline Hacinamiento & $0,56^{*}$ & 0,32 & $0,56^{*}$ \\
\hline \multicolumn{4}{|l|}{ NO UDI } \\
\hline Desempleo $^{1}$ & $0,82 *$ & $0,58^{*}$ & $0,83^{*}$ \\
\hline Instrucción Insuficiente $^{1}$ & $0,71^{*}$ & $0,64^{*}$ & $0,74 *$ \\
\hline Pobreza Extrema & $0,67 *$ & $0,73 *$ & $0,72 *$ \\
\hline Conflictividad social $^{1}$ & $0,79^{*}$ & $0,70^{*}$ & $0,81 *$ \\
\hline Hacinamiento & $0,47^{*}$ & $0,50^{*}$ & $0,52 *$ \\
\hline
\end{tabular}

UDIs: Usuarios de drogas por vía intravenosa.

${ }^{1}$ Estandarizado por edad

${ }^{*} \mathrm{p}<0,05$ correlacionada con el desempleo $\left(r_{\mathrm{s}}=0,83\right)$ y la conflictividad social $\left(r_{s}=0,81\right)$. Aunque en menor grado, la pobreza extrema muestra a su vez una importante correlación con la incidencia de TB en UDIs y en no UDIs. Las mujeres presentan en general $r_{s}$ menores a las de los hombres. En las mujeres no UDIs la correlación más importante se da con el indicador de pobreza extrema $\left(r_{s}=0,73\right)$ y de conflictividad social $\left(r_{\mathrm{s}}=0,70\right)$.

En el análisis multivariado del colectivo UDI, los hombres y mujeres presentaron un comportamiento similar, por lo que se pudo obtener un modelo multivariado conjunto que muestra además las diferencias de riesgo entre ambos sexos. Las variables asociadas a un mayor riesgo de TB de forma estadísticamente significativa fueron: el sexo, presentando mayor riesgo los hombres $(\mathrm{RR}=7,42)$, la edad (RR de los mayores de

Tabla 3

Asociación multivariada (regresión de Poisson) entre la tasa de incidencia de tuberculosis y los indicadores sociales en los barrios. Colectivos de UDIs y no UDIs según sexo. Barcelona 1990-1995

\begin{tabular}{|lrc|}
\hline & $R R$ & $I C 95 \%$ \\
\hline UDIs. Ambos sexos & & \\
Mayores 24 años & 61,51 & $(34,64-109,20)$ \\
Hombres & 7,42 & $(6,16-8,93)$ \\
Desempleo & 1,68 & $(1,51-1,88)$ \\
Conflictividad Social & 1,29 & $(1,04-1,58)$ \\
Hacinamiento & 1,36 & $(1,19-1,55)$ \\
Barrios de zona centro & 1,92 & $(1,48-2,50)$ \\
& & \\
No UDIs. Hombres & & \\
Desempleo en jóvenes & 1,11 & $(1,08-1,135)$ \\
Desempleo en mayores de 35 años & 1,15 & $(1.12-1,18)$ \\
Pobreza Extrema & 1,39 & $(1,17-1,65)$ \\
Barrios de zona centro & 1,80 & $(1,51-2,14)$ \\
& & \\
No UDIs. Mujeres & & \\
Menores 35 años & 1,29 & $(1,05-1,57)$ \\
Desempleo & 1,18 & $(1,09-1,26)$ \\
Pobreza extrema & 1,95 & $(1,56-2,45)$ \\
Barrios de zona centro & 1,72 & $(1,35-2,19)$ \\
\hline
\end{tabular}

UDIs: Usuarios de drogas por vía intravenosa.

RR: riesgo relativo; IC 95\%: Intervalo de confianza al 95\%. 
Asociación entre la tasa de incidencia anual media de TB que son UDIs y de TB no UDIs estandarizada por edad por 100.000 habitantes en los barrios de Barcelona, y diferentes indicadores sociales. Barcelona 1990-1995

Usuarios de drogas por vía intravenosa (UDIs)

Tasa de incidencia estandarizada por edad

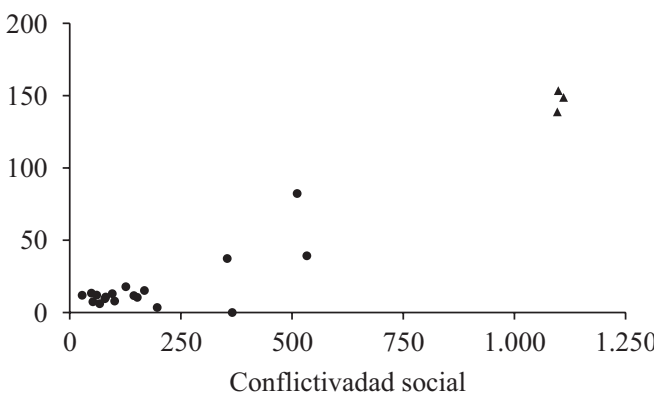

Tasa de incidencia estandarizada por edad

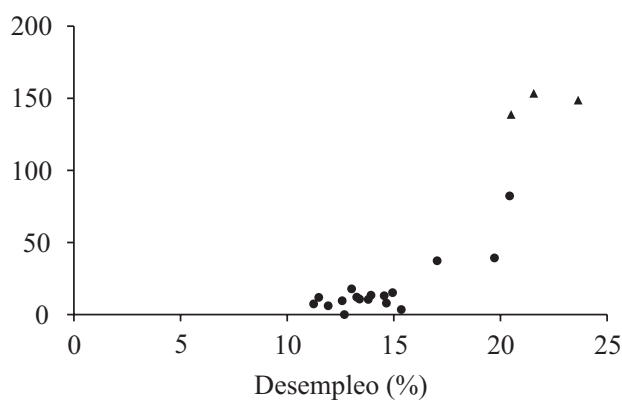

No usuarios de drogas por vía intravenosa (No UDIs)

Tasa de incidencia estandarizada por edad

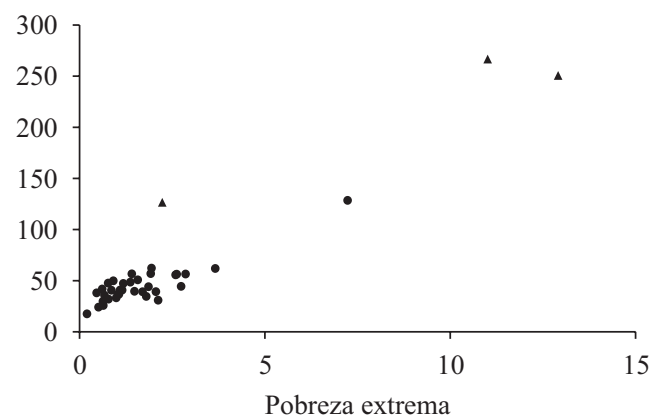

Tasa de incidencia estandarizada por edad

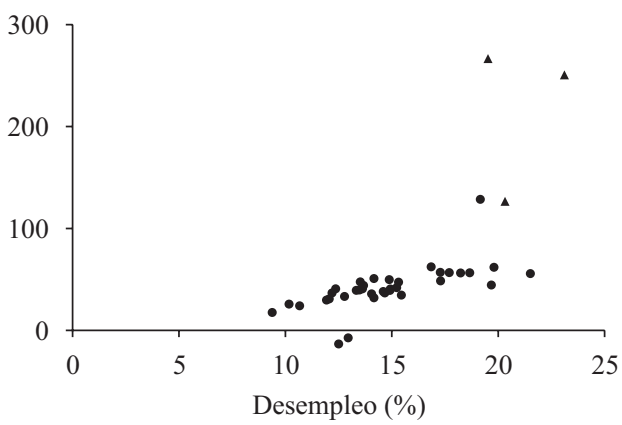

rs: Coeficiente de correlación de Spearman; $\mathbf{\Delta}$ : Barrios que presentan el fenómeno de zona centro; $\bullet$ Resto de barrios; Conflictividad Social: Tasa de personas encarceladas en prisión preventiva por 100.000 habitantes (estandarizada por edad); Pobreza extrema: Tasa de Pensiones Mínimas de reinserción por 1.000 habitantes; Desempleo: Porcentaje de habitantes en paro estandarizado por edad.

24 años $=61,5)$, el desempleo $(\mathrm{RR}=1,68)$, la conflictividad social $(\mathrm{RR}=1,29)$, el hacinamiento $(\mathrm{RR}=1,36)$ y el fenómeno de zona centro $(\mathrm{RR}=1,92)($ tabla 3$)$.

En los hombres no UDIs se detectó una interacción estadísticamente significativa en el modelo multivariado entre el desempleo y la edad: el riesgo de TB se asoció de forma estadísticamente significativa al desempleo en los jóvenes $(\mathrm{RR}=1,11)$ pero este riesgo aumentaba en los mayores de 35 años
$(\mathrm{RR}=1,15)$, incluso después de ajustar por los barrios con indicadores socioeconómicos más desfavorables (fenómeno de zona centro $\mathrm{RR}=1,80$ ). Los barrios con pobreza extrema presentaron un riesgo de TB 1,39 veces superior al de los barrios donde este indicador era más favorable. En las mujeres no UDIs, las menores de 35 años mostraron un riesgo de TB 1,29 veces superior comparadas con las mayores de 34 años. El desempleo y la pobreza extrema se asociaron también a un mayor riesgo de $\mathrm{TB}(\mathrm{RR}=1,18 \mathrm{y}$ 
$\mathrm{RR}=1,95$ ). Los barrios que presentan el fenómeno de zona centro muestran un riesgo de TB añadido con respecto al resto de barrios de la ciudad $(\mathrm{RR}=1,72)$ (tabla 3$)$.

\section{Validación del barrio de residencia}

En la tabla 4 se presentan los resultados del estudio de validación del barrio de residencia del registro de TB. De los 795 casos detectados durante 1995, en 780 (98\%) se pudo determinar si estaban o no empadronados. Los barrios con mayor proporción de casos de TB no empadronados fueron los del centro histórico de la ciudad, llegando hasta un 33,8\% en el caso del barrio del Ra- val. Los barrios con mayor proporción de casos de TB empadronados en un barrio diferente al que consta en el registro de TB fueron también los más céntricos, con porcentajes de hasta un 11,9\% (barrio del Parc). En el resto de barrios de la ciudad de Barcelona la proporción de individuos empadronados en el mismo barrio que figura en el registro de TB fue del $80,7 \%$, con sólo $13 \%$ individuos no empadronados y $6,3 \%$ individuos empadronados en un barrio diferente. Los porcentajes de casos con zona de residencia concordante entre los dos registros fue diferente de forma estadísticamente significativa en las diferentes áreas auditadas.

Tabla 4

Concordancia entre la variable barrio de residencia de los casos del registro de tuberculosis y la del Padrón Municipal de Habitantes. Barcelona, 1995

\begin{tabular}{|c|c|c|c|c|c|c|c|c|}
\hline \multirow{3}{*}{ Barrios del registro de TB } & \multicolumn{8}{|c|}{ Casos del registro de $T B$} \\
\hline & \multicolumn{2}{|c|}{ Concordantes $*$} & \multicolumn{2}{|c|}{ No empadronados } & \multicolumn{2}{|c|}{ Barrio diferente $* *$} & \multicolumn{2}{|c|}{ Total } \\
\hline & $N$ & $(\%)$ & $N$ & $(\%)$ & $N$ & $(\%)$ & $N$ & $\%$ \\
\hline \multicolumn{9}{|l|}{ Centro histórico } \\
\hline Barceloneta & 20 & $(74,1)$ & 6 & $(22,2)$ & 1 & $(3,7)$ & 27 & $(100)$ \\
\hline Parc & 31 & $(73,8)$ & 6 & $(14,3)$ & 5 & $(11,9)$ & 42 & $(100)$ \\
\hline Gòtic & 20 & $(58,8)$ & 11 & $(32,4)$ & 3 & $(8,8)$ & 34 & (100) \\
\hline Raval & 39 & $(54,9)$ & 24 & $(33,8)$ & 8 & $(11,3)$ & 71 & $(100)$ \\
\hline Resto de barrios & 489 & $(80,7)$ & 79 & $(13,0)$ & 38 & $(6,3)$ & 606 & (100) \\
\hline
\end{tabular}

* Concordantes: casos en que el barrio es igual al que consta en el Padrón de habitantes.

** Barrio diferente: casos en que el barrio no concuerda con el que consta en el Padrón de habitantes.

Significación de la prueba de Ji-cuadrado: $\mathrm{p}<0,001$.

\section{DISCUSIÓN}

En este estudio se ha evidenciado que en Barcelona la incidencia de TB, tanto de personas que son UDIs como de las que no lo son, es mayor en los cuatro barrios del centro histórico de la ciudad, caracterizados por tener bolsas de población marginal y elevada prevalencia de UDIs, seguido de algunos barrios de la periferia. El fenómeno de zona centro está muy asociado a la TB tanto en los UDIs como en los no UDIs. Además, los resultados muestran que los hombres, los mayores de 24 años, el desempleo, la conflictividad social y el hacinamiento están asociados a mayor riesgo de TB en el colectivo de los UDIs. La enfermedad en los no UDIs está asociada al desempleo y a la pobreza extrema. En los hombres, la asociación entre el desempleo y la TB es más importante en los mayores de 35 años; una posible explicación podría ser que el desem- 
pleo señala una privación social más importante en los mayores que en los jóvenes, ya que suele ser un desempleo de larga duración. De todos modos, es necesario señalar que a pesar de la existencia de una interacción estadísticamente significativa entre desempleo y edad en los hombres, la magnitud de las diferencias de los riesgos relativos entre los dos grupos de edad no es demasiado importante (RR de 1,11 y 1,15$)$.

Como en numerosos estudios anteriores, las dos variables más fuertemente asociadas con la TB en los UDIs son ser hombre y una mayor edad ${ }^{5,6}$. Por el contrario, las mujeres no UDIs menores de 35 años presentan un riesgo añadido de $\mathrm{TB}$, que puede indicar un perfil de mujeres no UDIs pareja de hombres UDIs, hipótesis que debería corroborarse con un estudio específico.

Anteriormente se han descrito desigualdades sociales en mortalidad en Barcelo$\mathrm{na}^{23,25,34}$, siendo las zonas con mayor privación socioeconómica donde existe un exceso de defunciones. Por otro lado, dichas desigualdades han aumentado en la década de los 90 a expensas del sida y la sobredosis a drogas de abuso ${ }^{24}$. Como podemos ver en este trabajo, estas desigualdades también se hacen patentes en la morbilidad, como en el caso que nos ocupa y en el de consumo de drogas ${ }^{7}$.

La relación entre la TB y la privación social, la pobreza extrema, el hacinamiento y la indigencia ha sido descrita en otros estudios. En el Reino Unido se ha enfatizado la importancia de tener en cuenta tanto la privación como la inmigración de países en que la TB es una enfermedad común; aunque la privación y la inmigración están relacionadas, ambos tienen efectos sobre la distribución geográfica de la TB, sobre todo en los años más recientes ${ }^{14,16,35,36}$. En Estados Unidos el resurgimiento de la TB se ha relacionado con la infección por VIH, las toxicomanías, el hacinamiento, la inmigración y la indigencia ${ }^{2}$. En los núcleos céntricos y pobres de las grandes ciudades del Este de
Estados Unidos, la combinación del uso de drogas por vía intravenosa, la concentración de pobreza, las personas «sin techo»y la infección por VIH, han tenido efectos adversos sobre todo en la población joven ${ }^{13}$. También las prisiones y los albergues de las personas «sin techo» han favorecido la transmisión ${ }^{6}$. Según un estudio de Jones et al, los factores predictivos de TB en casos de sida de Estados Unidos fueron: ser afroamericano o hispano, ser IDU y haber nacido fuera del país, presentando menos riesgo el colectivo de homosexuales ${ }^{37}$.

El presente estudio pone también en evidencia la asociación entre la TB y la pobre$\mathrm{za}$, siendo probablemente el VIH un factor desencadenante entre aquellos colectivos más desfavorecidos socio-económicamente. Este hecho se apoya en la menor frecuencia de TB en el colectivo de homosexuales no UDIs con sida, colectivo que probablemente tiene un mayor nivel socioeconómico ${ }^{37,38}$.

En nuestro estudio ha sido de gran utilidad el analizar separadamente la TB de UDIs y la TB de personas que no lo son, ya que presentan características diferenciales. La distribución de la TB de los UDIs está muy relacionada con la distribución de los UDIs en Barcelona. El colectivo de usuarios de drogas por vía parenteral está formado fundamentalmente por hombres de entre 25 y 35 años y se encuentra preferentemente en la zona centro de la ciudad y en las zonas con elevada conflictividad social ${ }^{7,34}$. Sin duda, la alta prevalencia de VIH en esta población hace que aumente la TB. Estos hechos se reflejan sobre todo en la relación entre la TB y la conflictividad social en el colectivo de UDIs. Por otro lado, hay que mencionar que el encarcelamiento también implica hacinamiento, lo que favorece la transmisión de la enfermedad y podría explicar un aumento de la incidencia en los barrios con mayor tasa de encarcelamiento.

Respecto a la medida de conflictividad social, hemos de señalar que hubiera sido interesante poder disponer de la tasa de en- 
carcelamiento de las mujeres y de los jóvenes, pero ésta es una información que no estaba disponible, así como otras variables que hubieran resultado de interés para el estudio, como puede ser la tasa de delitos.

El hecho de que en las personas tuberculosas que no son UDIs destaque la asociación con la pobreza extrema y el desempleo y no con la conflictividad social y el hacinamiento, nos muestra el patrón diferencial de este colectivo. De todos modos, es necesario no perder de vista que una limitación de este estudio es la medida del hacinamiento, ya que es una variable obtenida a nivel ecológico y, por lo tanto, no representa el grado de hacinamiento individual de las personas; además la definición utilizada puede no reflejar lo mismo que en estudios realizados en otros ámbitos. Aun y así, esta medida es la más utilizada en la bibliografía anglosajona, que es la más abundante sobre desigualdades en TB ${ }^{12,19}$. Probablemente hubiera sido mejor utilizar los metros cuadrados por persona, pero estos datos no estaban disponibles en el censo de población.

Asimismo es importante señalar que la privación material (desempleo) sigue estando relacionada con la TB una vez se ha tenido en cuenta el fenómeno de zona centro y, por lo tanto, los resultados obtenidos no se deben a unos pocos barrios con valores extremos, que se presentan muy alejados del resto tal como se observa en la figura 1 , si no que representaría un riesgo añadido para la TB.

En los modelos multivariados se ha usado el desempleo y no el nivel de instrucción ya que ambas variables están muy correlacionadas, y debido a que el desempleo se relaciona mejor con los indicadores de salud y su significado está más cercano al concepto de privación material ${ }^{39}$. Además, el nivel de instrucción tiene un efecto cohorte importante siendo las personas mayores las que tienen menos nivel, lo que no siempre refleja más privación material.
Las TEIs pueden estar sobrestimadas en los barrios en los que haya mayor proporción de individuos en situación irregular, no empadronados o empadronados en un barrio diferente al que viven, puesto que estos casos incrementarán el numerador y no el denominador (que sólo incluye la población empadronada en dicho barrio) de las tasas. Tal y como muestra el subestudio de validación de la variable barrio de residencia, los individuos residentes en los barrios con mayores incidencias de TB, que son los barrios que presentan indicadores sociales más desfavorables, con relativa frecuencia no se hallan empadronados o lo están en un barrio diferente al de residencia (es decir, el barrio del registro de TB no concuerda con el que consta en el padrón), y esto implica que las tasas en estos barrios podrían estar sobrestimadas con respecto al resto de barrios. Probablemente el hecho de que haya más casos en situación de empadronamiento irregular residentes en el casco antiguo, indica el efecto de atracción de personas vulnerables (desde el punto de vista socioeconómico y/o de la salud) y el proceso de desintegración social de los barrios que sufren el fenómeno de zona centro. De todos modos, la incidencia en los barrios más desfavorables es mucho más elevada que en el resto de (del orden de un $200 \%$ superior), incidencia que no estaría justificada sólo por las discrepancias en el empadronamiento.

Este estudio señala la presencia de desigualdades en la distribución de la TB en Barcelona que están relacionadas con distintos indicadores sociales y que son diferentes al tener en cuenta la TB de las personas UDIs y la TB de las que no son UDIs. Estos resultados pueden ser útiles para que se lleven a cabo intervenciones que tiendan a evitar estas desigualdades ${ }^{40}$. En el futuro sería interesante realizar un estudio de niveles múltiples incluyendo tanto variables de base individual como variables contextuales, para profundizar en las desigualdades sociales en TB. Para ello sería necesario mejorar la recogida de variables sociales en los registros de tuberculosis. 


\section{BIBLIOGRAFÍA}

1. World Health Organization: TB - A Global Emergency. Geneva: World Health Organization; 1994.

2. Snider DE. The new tuberculosis. N Eng J Med 1992;32:703-5.

3. Grupo de trabajo del PMIT. Incidencia de la tuberculosis en España: resultados del Proyecto Multicéntrico de Investigación en Tuberculosis (PMIT). Med Clin (Barc) 2000;114:530-537.

4. Caylà JA, García de Olalla P, Galdós-Tangüís H, Vidal R, López Colomés JLL, Gatell JM et al. The influence of Intravenous drug use and VIH infection in the transmission of tuberculosis. AIDS 1996; 10:95-100.

5. Caylà JA, Galdós-Tangüis H, Jansà JM, Olalla P, Brugal MT, Pañella E. Evolución de la Tuberculosis en Barcelona (1987-95). Influencia del virus de la inmunodeficiencia humana y de las medidas de control. Med Clin (Barc) 1998;111:608-615.

6. Selwyn PA. Tuberculosis and aids: epidemiologic, clinical and social dimensions. J Law Medicine Ethics 1993;21:279-87.

7. Brugal MT, Domingo-Salvany A, Maguire A, Caylà JA, Villalbí JR, Hartnoll RA small area analysis estimating the prevalence ofd addiction to opioids in Barcelona, 1993. J Epidemiol Community Health 1999; 53:488-494.

8. Wallace R. Urban desertification, public health and public order: «Planned shrinkage», violent death, substance abuse and aids in the Bronx. Soc Sci Med 1990, 31: $801-813$

9. Simon PA. Income and aids rates in Los Angeles County. AIDS 1995;9:281-4.

10. Lowinger P. Drug abuse: economic and political basis. En: Lowinson $\mathrm{JH}$, Ruiz P, Millman R. Substance abuse. A comprehensive textbook. Baltimore: Williams\&Wilkins, 1992:138-143

11. Wilkinson J. Problematic Drug Use and Social Deprivation. Public Health, 1987; 101:165-168.

12. Spence DP, Hotchkiss J, Williams CS, Davies PD Tuberculosis and poverty. BMJ 1993;307:759-761.

13. Perlman DC, Salomon N, Perkins MP, Yancovitz, Paone D, Jarlais DDJ. Tuberculosis in drug users. Clinical Infectious Diseases 1995;21:1253-64.

14. Mangtani P. Socioeconomic deprivation and notification rates for tuberculosis in London during 1982 - 91. BMJ 1995; 310:963-966.
15. Darbyshire JH. Tuberculosis: old reasons for a new increase? BMJ 1995;310:954-955.

16. Barkham TMS, Drury A, Pearson AD, Dybowsk $\mathrm{R}$, Atkinson H. Tuberculosis in inner London: evidence for an increase in young adults and immigrants. Epidemiol Infect 1995;115:133-137.

17. Elender F, Bentham G, Langford I. Tuberculosis mortality in England and Wales during 1982-1992: its association with poverty, ethnicity and aids. Soc Sci Med 1998;46:673-681.

18. Hawker JI, Bakhshi SS, Ali S, Farrington AP. Ecological analysis of ethnic differences in relation between tuberculosis and poverty. BMJ 1999;319:1031-1034

19. Drucker E, Alcabes P. Childhood tuberculosis in the Bronx, New York. Lancet 1994;343:1482-85.

20. Barnes PF. Transmission of tuberculosis among the urban homeless. JAMA 1996; 275:305-7.

21. Layton MC. Tuberculosis screening among homeless persons with aids living in single-room-occupancy hotels. Am J Public Health 1995;85:1556-8.

22. Barnes PF. Tuberculosis among the inner city poor. Int J Tuberc Lung Dis 1998;2(9): S41-S45.

23. Borrell C, Arias A. Socioeconomic factors and mortality in urban settings: the case of Barcelona, Spain. J Epidemiol Comm Health 1995;49:460-65.

24. Borrell C, Plasencia A, Pasarín M, Ortún V. Widening social inequalities in mortality: the case of Barcelona, a southern Europen city. J Epidemiol Community Health 1997;51:659-667.

25. Pasarín I, Borrell C, Plasència A. ¿Dos patrones de desigualdades sociales en mortalidad en Barcelona? Gac Sanitaria 1999; 13:431-440.

26. Solsona J, Cayla JA, Verdu E, Estrada MP, Garcia S, Roca D, et al. Molecular and conventional epidemiology of tuberculosis in an inner city district. Int J Tuberc Lung Dis. 2001 Aug;5(8):724-31.

27. Padrón de Habitantes de la ciudad de Barcelona 1991. Servicio de Estadística. Barcelona: Ayuntamiento de Barcelona; 1992.

28. Generalitat de Catalunya. Institut d'Estadística de Catalunya. Cens d'Habitants, 1991.

29. Rué M, Borrell C. Los métodos de estandarización de tasas. Revisiones en Salud Pública 1993;3:263-95.

Rev Esp Salud Pública 2001, Vol. 75, N. ${ }^{\circ} 6$ 
30. Morgenstern H. Ecologic studies in epidemiology: concepts, principles, and methods. Annu Rev Public Health. 1995; 16:61-81.

31. Breslow NE, Day NE. Statistical methods in cancer research. Volume II: The design and analysis of cohort studies. Lyon: International Agency for Research in Cancer; 1987.

32. Marija J. Norusis / SPSS Inc. SPSS for Windows Advanced Statistics, Release 5; 1992.

33. EGRET Reference Manual, First Draft. 1990.

34. Torralba L1, Brugal MT, Villalbí JR, Toribio A, Valverde JL, Tortosa MT. Mortality due to adverse drug reactions: opiates and cocaine in Barcelona 1989-93. Addiction 1996;91:419-426

35. Tocque K, Regan M, Remmington T, Beeching NJ, Jamienson I, Syed Q et al Social factors associated with increases in tuberculosis notifications. Eur Respir J 1999;13:541-545.
36. Tocque K, Doherty MJ, Bellis MA, Spence DPS, Williams CSD, Davies PDO. Tuberculosis notifications in England: the relative effects of deprivation and immigration. Int $J$ Tuberc Dis 1998;2(3):213-128.

37. Jones JF, Burwen DR, Fleming PL, Ward JW. Tuberculosis among AIDS patients in the United States, 1993. J AIDS Hum Retrovirol 1996;12:293-297.

38. García de Olalla $\mathrm{P}$, Caylà JA, Jansà JM, Brugal MT, Maldonado R, Pañella H. Sida en Barcelona, vigilancia epidemiológica. Informe trimestral 49. Barcelona: Ayuntamiento de Barcelona; 2000.

39. Smith GD, Hart C, Hole D, McKinnon P, Gillis $\mathrm{C}$, Watt $\mathrm{G}$ et al. Education and occupational social class: wich is the more important indicator of mortality risk? J Epidemiol Community Health 1998;52:153-160.

40. Zumla A, Grange JM. Doing something about tuberculosis. BMJ 1999;318:956. 